\title{
Two sides of the coin
}

\begin{abstract}
Aging parents transmit an increasing burden of chromosomal aberrations and mutations. There are also epidemiological correlations between parental age and neurodevelopmental disorders, including autism. The citation and interpretation of these two lines of evidence should be carefully evaluated.
\end{abstract}

B $y$ focusing specifically on the mutational processes prevalent in chromosomes of paternal origin, we may be able to explain some fraction of neurodevelopmental disorders, but we must not forget the epidemiological evidence for effects of the age of both parents on the incidence of autism, which may point to the impact of other genetic factors as well as gestational and environmental influences.

Several studies reporting only the increase in autism with increasing paternal age (Arch. Gen. Psychiatry 63, 1026-1032, 2006, and Mol. Psychiatry 16, 1203-1212, 2010) are widely cited in discussion. However, the weight of the epidemiological evidence shows that there is a relatively modest increase in the incidence of autism independently associated with maternal and paternal age. Grether et al. (Am. J. Epidemiol. 170, 1118$1126,2009)$ reported an odds ratio for autism of 1.38 (95\% confidence interval 1.32-1.44) for a ten-year increase in maternal age and $1.22(1.18-1.26)$ for a ten-year increase in paternal age on the basis of analysis of 23,311 children with autism from a cohort of 7,550,026 births. These results confirm the original findings from the analysis of 593 children with autism from 150,414 births (Arch. Pediatric Adolesc. Med. 161, 334-340, 2007). Meta-analysis of 16 studies resulted in a similar conclusion (J. Am. Acad. Child Adolesc. Psychiatry 51, 477-486, 2012), namely that autism risk of a similar magnitude is associated with maternal age and remains associated, even after correction for the correlation between the ages of the two parents.

The authors of recent autism genomics publications vary in the boldness with which they relate the role of parental mutation rate to neurodevelopmental phenotypes, reflecting not only their data but the range of hypotheses under consideration. O'Roak et al. (Nature 485, 246-250, 2012) found a 4:1 ratio of paternal to maternal de novo point mutations in 677 individual exomes from 209 families with autism spectrum disorders (ASD). They state that this difference in parental origin and the correlation of de novo variant frequency with paternal age are "consistent with the modest increased risk for children of older fathers to develop ASD."
Kong et al. (Nature 488, 471-475, 2012) analyzed mutation rates across the genomes of 78 trios and found by multiple regression that the mutational effect of parental age is only significant for the paternal contribution (at 2.01 mutations per year). Sanders et al. (Nature 485, 237-241, 2012) examined the exomes of 238 pedigrees with an affected proband and found an increase in de novo single-nucleotide variations (SNVs) with paternal age, noting the strong correlation between paternal and maternal age. By comparing coding SNVs in 200 sibling pairs discordant for ASD, these authors estimated that at least $14 \%$ of individuals with ASD in the Simons Simplex collection carry de novo SNVs influencing autism risk. Neale et al. (Nature $485,242-245,2012)$ reported that both maternal and paternal age strongly influenced the number of de novo nonsense and missense SNVs in the exomes of 175 ASD probands, but there was no evidence for paternal excess. An elevated ratio of paternal to maternal mutations was also found for SNVs in a single pedigree (Nat. Genet. 44, 1277-1281, 2012) and for microsatellites (Nat. Genet. 44, 1161-1165, 2012). These findings have recently been reviewed by Matthew Hurles (Nat. Genet. 44, 1174-1176, 2012).

The maternal age contribution to autism does not need to be entirely genetic. Indeed, research into environmental influences has shown some resurgence since the twin-based heritability estimate for autism was revised downward by Hallmayer et al. (Arch. Gen. Psychiatry 68, 1095-1102, 2011). Sequencing studies aimed at determining the heritable and de novo mutational burden and specific mutational patterns will in the foreseeable future be smaller and more prone to bias than epidemiological studies and typically address the readily interpretable genetic component of neurodevelopmental disorders. Not all evidence implicating genetic variants in complex conditions will be consistent with a single mechanism. While we are trying to find all of the causal influences, journals and referees need to give authors space and encouragement to represent the supporting evidence in a full and skeptical way, lest we miss an opportunity to understand the mechanisms of developmental disabilities. 